\title{
Sufficient Fritz John Type Optimality Criteria and Duality for Control Problems
}

\author{
Iqbal Husain, Santosh K. Srivastav
}

Department of Mathematics, Jaypee University of Engineering and Technology, Guna, India.

Email: ihusain11@yahoo.com, santosh_00147@rediffmail.com

Received December $17^{\text {th }}, 2012$; revised January $18^{\text {th }}$, 2013; accepted February $18^{\text {th }}, 2013$

Copyright (C 2013 Iqbal Husain, Santosh K. Srivastav. This is an open access article distributed under the Creative Commons Attribution License, which permits unrestricted use, distribution, and reproduction in any medium, provided the original work is properly cited.

\begin{abstract}
Sufficient Fritz John optimality conditions are obtained for a control problem in which objective functional is pseudoconvex and constraint functions are quasiconvex or semi-strictly quasiconvex. A dual to the control problem is formulated using Fritz John type optimality criteria instead of Karush-Kuhn-Tucker optimality criteria and hence does not require a regularity condition. Various duality results amongst the control problem and its proposed dual are validated under suitable generalized convexity requirements. The relationship of our duality results to those of a nonlinear programming problem is also briefly outlined.
\end{abstract}

Keywords: Sufficient Optimality Criteria; Control Problem; Fritz John Type Dual; Nonlinear Programming Problem; Generalized Convexity

\section{Introduction}

Optimal control models represent a variety of common situations, notably, advertising investment, production and inventory, epidemic, control of a rocket, etc. The optimal planning of a river system which is an invincible resource of nature, where it is needed to make the best use of the water, can also be modelled as an optimal control problem. Optimal control models are also potentially applicable to economic planning and to the world models of the "Limits to Growth" kind in general.

Optimality criteria for any optimization problem are of great significance and lay the foundation of the concept of duality. Fritz John optimality criteria for a control problem were first derived by Berkovitz [1]. Subsequently Mond and Hanson [2], who first investigated duality in optimal control pointed out that from Fritz John optimal criteria, Karush-Kuhn-Tucker optimality criteria can be deduced if normality of the solution of a control problem which replaces a regularity conditions is assumed. Later, treating a nondifferentiable control problem as a nondifferentiable mathematical programming problem in an infinite-dimensional space, Chandra et al. [3], obtained Fritz John as well as Karush-Kuhn-Tucker optimality criteria.

For a nondifferentiable control problem Using KarushKuhn-Tucker optimality criteria, they formulated Wolfe type dual and derived usual duality results under appropriate convexity assumptions.

In this research exposition, sufficient Fritz John criteria are derived for a differentiable control problem in which objective functional is pseudoconvex and constraint functions are quasiconvex or semi-strictly pseudoconvex. A number of duality results are proved for relating the solution of the control problem with that of its proposed dual under suitable generalized convexity requirements. The relationship of our duality results to those of a nonlinear programming problem is indicated.

\section{Control Problem and Related Preliminaries}

Let $R^{n}$ denotes a n-dimensional Euclidean space, $I=[a, b]$ be a real interval and $f: I \times R^{n} \times R^{m} \rightarrow R$ be a continuously differentiable with respect to each of its arguments. For the function $f(t, x, u)$ where

$x: I \rightarrow R^{n}$ is differentiable with its derivative $x$ and $u: I \rightarrow R^{m}$ is the smooth function, denote the partial derivatives of $f$ by $f_{t}, f_{x}$ and $f_{u}$, where

$$
\begin{aligned}
& f_{t}: \frac{\partial f}{\partial t}, f_{x}=\left(\frac{\partial f}{\partial x^{1}}, \quad, \frac{\partial f}{\partial x^{n}}\right)^{\mathrm{T}}, f_{u}=\left(\frac{\partial f}{\partial u^{1}}, \quad, \frac{\partial f}{\partial u^{m}}\right)^{\mathrm{T}}, \\
& x=\left(x^{1}, \quad, x^{n}\right)^{\mathrm{T}} \text { and } u=\left(u_{1}, \quad, u_{m}\right)^{\mathrm{T}} .
\end{aligned}
$$


For $m$-dimensional vector function $g(t, x, u)$ the gredient with respect to $x$ is

$$
g_{x}=\left(\begin{array}{ll}
\frac{\partial g^{1}}{\partial x^{1}}, & , \frac{\partial g^{p}}{\partial x^{n}} \\
\frac{\partial g^{1}}{\partial x^{n}}, & , \frac{\partial g^{p}}{\partial x^{n}}
\end{array}\right),
$$

a $n \times p$ matrix of first order derivatives.

Here $u(t)$ is the control variable and $x(t)$ is the state variable, $u$ is related to $x$ via the state equation $x=h(t, x, u)$. Gradients with respect to $u$ are defined analogously.

A control problem is to transfer the state vector from an initial state $x(a)=\alpha$ to a final state $x(b)=\beta$ so as to minimize a functional, subject to constraints on the control and state variables.

A control problem can be stated formally as,

(CP): $\underset{x \in X, u \in U}{\operatorname{Minimize}} \int_{a}^{b} f(t, x, u) \mathrm{d} t$, subject to

$$
\begin{gathered}
x(a)=\alpha, x(b)=\beta \\
h(t, x, u)=x, t \in I \\
g(t, x, u) \leq 0, t \in I
\end{gathered}
$$

1) $f$ is as before, $g: I \times R^{n} \times R^{m} \rightarrow R^{p}$ and $h: I \times R^{n} \times R^{m} \rightarrow R^{n}$ are continuously differentiable functions with respect to each of its arguments.

2) $X$ is the space of continuously differentiable state functions $x: I \rightarrow R^{n}$ such that $x(a)=\alpha, x(b)=\beta$ equipped with the norm $\|x\|=\|x\|_{\infty}+\|D x\|_{\infty}$, and $u$ is the space of piecewise continuous control functions $u: I \rightarrow R^{m}$ has the uniform norm $\|\cdot\|_{\infty}$ and

3) The differential Equation (2) for $x$ with the initial conditions expressed as

$x(t)=x(a)+\int_{a}^{t} h(s, x(s), u(s)) \mathrm{d} s, t \in I$, may be written as $D x=H(x, u)$, where the map

$H: X \times U \rightarrow C\left(I, R^{n}\right), C\left(I, R^{n}\right)$ being the space of continuous functions from $I \rightarrow R^{n}$, defined by

$H(x, u)=h(t, x(t), u(t))$.

Following Craven [4], the control problem can be expressed as,

(ECP): $\underset{x \in X, u \in U}{\operatorname{Minimize}} F(x, u)$ subject to

$$
\begin{aligned}
& D x=H(x, u), \\
& -G(x, u) \in S,
\end{aligned}
$$

where $G$ is function from $X \times U$ into $C\left(I, R^{p}\right)$ given by $G(x, u)(t)=g(t, x(t), u(t))$ from

$x \in X, u \in U$, and $t \in I ; S$ is the convex cone of func- tions in $C\left(I, R^{p}\right)$ whose components are non-negative; thus $S$ has interior points.

Necessary optimality conditions for existence of extermal solution for a variational problem subject to both equality and inequality constraints were given by valentine [5]. Invoking Valentine’s [5] results, Berkovitz [1] obtained corresponding necessary optimality criteria for the above control problem (CP). Here we state the Fritz John type optimality conditions derived by Chandra et al. [3] in of the following proposition which will be required in the sequel.

\section{Proposition 1}

\section{(Necessary Optimality Conditions)}

If $(\bar{x}, \bar{u}) \in X \times U$ an optimal solution of (CP) and the Frechet derivatives $Q^{\prime}=\left(D-H_{x}(x, u)-H_{u}(x, u)\right)$ is surjective, then there exist Lagrange multipliers $r \in R$, and piecewise smooth functions $y: I \rightarrow R^{p}$ and $z: I \rightarrow R^{n}$ satisfying, for all $t \in I$,

$$
\begin{gathered}
r f_{x}(t, \bar{x}, \bar{u})+y(t)^{\mathrm{T}} g_{x}(t, \bar{x}, \bar{u}) \\
+z(t)^{\mathrm{T}} h_{x}(t, \bar{x}, \bar{u})+z(t)=0, t \in I \\
r f_{u}(t, \bar{x}, \bar{u})+y(t)^{\mathrm{T}} g_{u}(t, \bar{x}, \bar{u}) \\
+z(t)^{\mathrm{T}} h_{u}(t, \bar{x}, \bar{u})=0, t \in I \\
y(t)^{\mathrm{T}} g(t, \bar{x}, \bar{u})=0, t \in I \\
(r, y(t)) \geq 0, t \in I \\
(r, y(t), z(t)) \neq 0, t \in I
\end{gathered}
$$

The above conditions will become Karush-Kuhn-Tucker conditions if $r>0$. Therefore, if we assume that the optimal solutions $(\bar{x}, \bar{u})$ is normal, then without any loss of generality, we can set $r=1$. Thus from the above we have the Karush-Kuhn-Tucker type optimality conditions

$$
\begin{aligned}
& f_{x}(t, \bar{x}, \bar{u})+y(t)^{\mathrm{T}} g_{x}(t, \bar{x}, \bar{u}) \\
& +z(t)^{\mathrm{T}} h_{x}(t, \bar{x}, \bar{u})+z(t)=0, t \in I, \\
& f_{u}(t, \bar{x}, \bar{u})+y(t)^{\mathrm{T}} g_{u}(t, \bar{x}, \bar{u}) \\
& +z(t)^{\mathrm{T}} h_{u}(t, \bar{x}, \bar{u})=0, t \in I, \\
& y(t)^{\mathrm{T}} g(t, \bar{x}, \bar{u})=0, t \in I, \\
& y(t) \geq 0, t \in I .
\end{aligned}
$$

Using these optimality conditions, Mond and Hanson [2] constructed following Wolfe type dual.

$$
\begin{aligned}
\text { Maximize } \int_{a}^{b}\left\{f(t, x, u)+y(t)^{\mathrm{T}} g(t, x, u)\right. \\
\left.+z(t)^{\mathrm{T}}(h(t, x, u)-x)\right\} \mathrm{d} t
\end{aligned}
$$


subject to

$$
\begin{aligned}
& f_{x}(t, x, u)+y(t)^{\mathrm{T}} g_{x}(t, \bar{x}, \bar{u}) \\
& +z(t)^{\mathrm{T}} h_{x}(t, x, u)+z(t)=0, t \in I \\
& f_{u}(t, x, u)+y(t)^{\mathrm{T}} g_{u}(t, \bar{x}, \bar{u}) \\
& +z(t)^{\mathrm{T}} h_{u}(t, x, u)=0, t \in I, y(t) \geq 0, t \in I
\end{aligned}
$$

In [6], [CP] and (CD) are shown to from a dual pair if $f$, $g$ and $h$ are all convex in $x$ and $u$. Subsequently, Mond and Smart [6] extended this duality under generalized invexity.

As a follows up, Husain et al. [7] formulated the following dual (CD) to the primal problem (CP) in the spirit of Mond and Weir [8].

(CD): Maximize $\int_{a}^{b} f(t, x, u) \mathrm{d} t$

subject to

$$
\begin{aligned}
& x(a)=\alpha, x(b)=\beta \\
& f_{x}(t, x, u)+y(t)^{\mathrm{T}} g_{x}(t, x, u) \\
& +z(t)^{\mathrm{T}} h_{x}(t, x, u)+z(t)=0, t \in I \\
& f_{u}(t, x, u)+y(t)^{\mathrm{T}} g_{u}(t, x, u) \\
& +z(t)^{\mathrm{T}} h_{u}(t, x, u)=0, t \in I \\
& \int_{a}^{b}\left(y(t)^{\mathrm{T}} g(t, x, u)+z(t)^{\mathrm{T}}(h(t, x, u)-x)\right) \mathrm{d} t \geq 0, \\
& y(t) \geq 0, t \in I .
\end{aligned}
$$

They proved sufficiency of the optimality criteria and duality for the pair of dual problems (CP) and (CD) under pseudoinvexity of $\int \mathrm{d} t$ and quasi-invexity of $\int_{a}^{b}\left(\bar{y}^{\mathrm{T}} g+\bar{z}^{\mathrm{T}}(h-x)\right) \mathrm{d} t$.

\section{Sufficiency of Fritz Type Optimality Criteria}

Before proceeding to the main results of this section, we formulate the following definitions which will be required in the forthcoming analysis:

Definitions: 1) For $\phi: R^{n} \times R^{m} \times I \rightarrow R$, the functional $\int_{a}^{b} \phi \mathrm{d} t$ is said to be strict pseudoconvex, if all $(x, u) \neq(\bar{x}, \bar{u})$,

$$
\begin{aligned}
& \int_{a}^{b}\left\{(x-\bar{x}) \phi_{x}(t, \bar{x}, \bar{u})+(u-\bar{u}) \phi_{u}(t, \bar{x}, \bar{u})\right\} \mathrm{d} t \\
& \geq 0 \Rightarrow \int_{a}^{b} \phi(t, x, u) \mathrm{d} t>\int_{a}^{b} \phi(t, \bar{x}, \bar{u}) \mathrm{d} t
\end{aligned}
$$

Equivalently

$$
\begin{aligned}
& \int_{a}^{b} \phi(t, x, u) \mathrm{d} t \leq \int_{a}^{b} \phi(t, \bar{x}, \bar{u}) \mathrm{d} t \\
& \Rightarrow \int_{a}^{b}\left\{(x-\bar{x}) \phi_{x}(t, \bar{x}, \bar{u})+(u-\bar{u}) \phi_{u}(t, \bar{x}, \bar{u})\right\} \mathrm{d} t<0
\end{aligned}
$$

2) For $y: I \rightarrow R^{m}$, the functional $\int_{a}^{b} y^{\mathrm{T}} g \mathrm{~d} t$ is semistrictly pseudoconvex if $\int_{a}^{b} y^{\mathrm{T}} g \mathrm{~d} t$ is strictly pseudoconvex for all $y(t) \geq 0, y(t) \neq 0$.

If $\phi(t, x, u), y(t)^{\mathrm{T}}$ and $g(t, x, u)$ are independent of $t$ and $u$ then the above definitions reduce to those of [6].

Theorem 1 (Sufficiency): If $\int f \mathrm{~d} t$ is pseudoconvex, $\int_{I} \bar{y}(t)^{\mathrm{T}} g \mathrm{~d} t$ is semi-strictly pseudóconvex and $\int_{I} \bar{z}(t)^{\mathrm{T}}(h-x) \mathrm{d} t$ is quasiconvex, and if there exist $\bar{r} \in R$ and piecewise smooth $\bar{y}: I \rightarrow R^{m}$ and $\bar{z}: I \rightarrow R^{n}$ such that from (4)-(8) are satisfied, then $(\bar{x}, \bar{u})$ is an optimal solution of (CP).

Proof: Suppose that $(\bar{x}, \bar{u})$ is not optimal for (CP) i.e. there exist $(x, u) \neq(\bar{x}, \bar{u})$ such that

$$
\int_{I} f(t, x, u) \mathrm{d} t<\int_{I} f(t, \bar{x}, \bar{u}) \mathrm{d} t
$$

This, by pseudoconvexity of $\int f \mathrm{~d} t$ implies

$$
\int_{I}\left\{(x-\bar{x})^{\mathrm{T}} f_{x}(t, \bar{x}, \bar{u})+(u-\bar{u})^{\mathrm{T}} f_{u}(t, \bar{x}, \bar{u})\right\} \mathrm{d} t<0
$$

and

$$
\int_{I}\left\{(x-\bar{x})^{\mathrm{T}} r f_{x}(t, \bar{x}, \bar{u})+(u-\bar{u})^{\mathrm{T}} r f_{u}(t, \bar{x}, \bar{u})\right\} \mathrm{d} t \leq 0
$$

with strict inequality in (9) if $\bar{r}>0$.

Feasibility of $(x, u)$ for (CP) together with (6) implies,

$$
\int_{I} \bar{y}(t)^{\mathrm{T}} g(t, x, u) \mathrm{d} t \leq \int_{I} \bar{y}(t)^{\mathrm{T}} g(t, \bar{x}, \bar{u}) \mathrm{d} t
$$
which by semi-strict pseudoconvexity of $\int_{I} \bar{y}(t)^{\mathrm{T}} g \mathrm{~d} t$,
implies

$$
\begin{aligned}
& \int_{I}\left\{(x-\bar{x})\left(\bar{y}^{\mathrm{T}}(t) g_{x}(t, x, u)\right)\right. \\
& \left.+(u-\bar{u})\left(\bar{y}^{\mathrm{T}}(t) g_{u}(t, \bar{x}, \bar{u})\right)\right\} \mathrm{d} t \leq 0
\end{aligned}
$$

with strict inequality in (10) if some $y^{i}(t)>0, i \in\{1,2, \quad, m\}$.

Also

$$
\begin{aligned}
& \int_{I} \bar{Z}(t)^{\mathrm{T}}(h(t, x, u)-x) \mathrm{d} t \\
& \leq \int_{I} \bar{Z}(t)^{\mathrm{T}}(h(t, \bar{x}, \bar{u})-\bar{x}) \mathrm{d} t
\end{aligned}
$$


This, in view of quasiconvexity of $\int_{I} z(t)^{\mathrm{T}}(h-\bar{x}) \mathrm{d} t$ yields

$$
\begin{aligned}
& \begin{array}{r}
\geq \int_{I}\left\{(x-\bar{x})^{\mathrm{T}} \bar{z}(t)^{\mathrm{T}} h_{x}(t, \bar{x}, \bar{u})+(x-\bar{x}) z(t)\right. \\
\left.+(u-\bar{u}) z(t)^{\mathrm{T}} h_{u}(t, \bar{x}, \bar{u})\right\} \mathrm{d} t
\end{array} \\
& =\int_{I}\left\{(x-\bar{x})^{\mathrm{T}}\left(z(t)^{\mathrm{T}} h_{x}(t, \bar{x}, \bar{u})-\bar{z}(t)\right)\right. \\
& \left.+(u-\bar{u})^{\mathrm{T}} z(t)^{\mathrm{T}} h_{u}(t, x, u)\right\} \mathrm{d} t \\
& +\left.(x-\bar{x}) z(t)\right|_{t=a} ^{t=b}
\end{aligned}
$$

(By integrating by parts)

$$
\begin{array}{r}
=\int_{I}\left\{(x-\bar{x})^{\mathrm{T}}\left(z(t)^{\mathrm{T}} h_{x}(t, \bar{x}, \bar{u})-\bar{z}(t)\right)\right. \\
\left.+(u-\bar{u})^{\mathrm{T}} z(t)^{\mathrm{T}} h_{u}(t, x, u)\right\} \mathrm{d} t
\end{array}
$$

(Using (1))

Combining (9)-(11), we have

$$
\begin{gathered}
\int_{I}\left\{( x - \overline { x } ) ^ { \mathrm { T } } \left(r f_{x}(t, \bar{x}, \bar{u})+\bar{y}(t) g_{x}(t, \bar{x}, \bar{u})\right.\right. \\
\left.+\bar{z}(t) h_{x}(t, \bar{x}, \bar{u})-\bar{z}(t)\right) \\
+(u-\bar{u})\left(r f_{u}(t, \bar{x}, \bar{u})+\bar{y}(t) g_{u}(t, \bar{x}, \bar{u})\right. \\
\left.\left.+\bar{z}(t) h_{u}(t, \bar{x}, \bar{u})-\bar{z}(t) h_{u}(t, \bar{x}, \bar{u})\right)\right\} \mathrm{d} t<0
\end{gathered}
$$

This contradicts (4) and (5). Hence $(\bar{x}, \bar{u})$ is an optimal solution of (CP).

\section{Fritz Type Duality}

The following is the Fritz john type dual to the problem (CP):

$\left(\mathrm{F}_{\mathrm{r}} \mathrm{CD}\right)$ : Maximize $\int_{I} f(t, x, u) \mathrm{d} t$

subject to

$$
\begin{gathered}
x(a)=\alpha, x(b)=\beta \\
r f_{x}(t, x, u)+y^{\mathrm{T}}(t) g_{x}(t, x, u) \\
+z^{\mathrm{T}}(t) h_{x}-z(t)=0, t \in I \\
r f_{u}(t, x, u)+y^{\mathrm{T}}(t) g_{u}(t, x, u) \\
+z^{\mathrm{T}}(t) h_{u}=0, t \in I \\
\int_{I} y(t)^{\mathrm{T}} g(t, x, u) \mathrm{d} t \geq 0, \\
\int_{I} z(t)^{\mathrm{T}}(h(t, x, u)-x(t)) \mathrm{d} t \geq 0
\end{gathered}
$$

$$
\begin{gathered}
(r, y(t)) \geq 0, t \in I \\
(r, y(t), z(t)) \neq 0, t \in I
\end{gathered}
$$

Theorem 2 (Weak Duality): Assume that

$\left(\mathrm{A}_{1}\right)$ satisfies $(\bar{x}, \bar{u})$ is feasible for $(\mathrm{CP})$ and $(x, u, r, y, z)$ is feasible for $\left(\mathrm{F}_{\mathrm{r}} \mathrm{CD}\right)$.

$\left(\mathrm{A}_{2}\right): \int_{I} f(t, x, u) \mathrm{d} t$ is pseudo-convex,

$\int_{I} y(t)^{\mathrm{T}} g(t, x, u) \mathrm{d} t$ is semi-strictly pseudo-convex and

$\int_{I} z(t)^{\mathrm{T}}(h(t, x, u)-x) \mathrm{d} t$ is quasi-convex.

Then

$$
\inf (\mathrm{CP}) \geq \operatorname{Sup}\left(\mathrm{F}_{\mathrm{r}} \mathrm{CD}\right)
$$

Proof: Suppose $\int_{I} f(t, \bar{x}, \bar{u}) \mathrm{d} t<\int_{I} f(t, x, u) \mathrm{d} t$

This, because of pseudo-convexity of $\int_{I} f(t, x, u) \mathrm{d} t$ yields $\int_{I}\left\{(\bar{x}-x)^{\mathrm{T}} f_{x}(t, x, u)+(\bar{u}-u)^{\mathrm{T}} f_{u}(t, \bar{x}, \bar{u})\right\} \mathrm{d} t<0$ and

$$
\int_{I}\left\{(\bar{x}-x)^{\mathrm{T}} r f_{x}(t, x, u)+(\bar{u}-u)^{\mathrm{T}} r f_{u}(t, \bar{x}, \bar{u})\right\} \mathrm{d} t \leq 0
$$

with strict inequality in the above with $r>0$. From the constraints of (CP) and ( $\left.\mathrm{F}_{\mathrm{r}} \mathrm{CD}\right)$, we have

$$
\int_{I} y(t)^{\mathrm{T}} g(t, \bar{x}, \bar{u}) \mathrm{d} t \leq \int_{I} y(t)^{\mathrm{T}} g(t, x, u) \mathrm{d} t
$$

which by semi-strictly pseudo-convexity of $\int_{I} y(t)^{\mathrm{T}} g(\cdot) \mathrm{d} t$, implying

$$
\int_{I}\left\{(\bar{x}-x)\left(y(t)^{\mathrm{T}} g_{x}\right)+(\bar{u}-u)\left(y(t)^{\mathrm{T}} g_{u}\right)\right\} \mathrm{d} t \leq 0
$$

with strict inequality with $y_{i}(t)>0, t \in I$, $i \in\{1,2,3, \quad, m\}$. Also, we have

$$
\begin{aligned}
& \int_{I} z(t)^{\mathrm{T}}(h(t, \bar{x}, \bar{u})-\bar{x}) \mathrm{d} t \\
& \leq \int_{I} z(t)^{\mathrm{T}}(h(t, x, u)-x) \mathrm{d} t
\end{aligned}
$$

Using quasi-convexity of $\int_{I} z(t)^{\mathrm{T}}(h(t, x, u)-\bar{x}) \mathrm{d} t$ in the above, we have

$$
\int_{I}\left\{(\bar{x}-x)^{\mathrm{T}} h_{x}+(\bar{x}-x)^{\mathrm{T}} z(t)+(\bar{u}-u)^{\mathrm{T}} h_{u}\right\} \mathrm{d} t \leq 0
$$

which as earlier becomes

$$
=\int_{I}\left\{(\bar{x}-x)^{\mathrm{T}}\left(z h_{x}-z\right)+(\bar{u}-u)^{\mathrm{T}} z h_{u}\right\} \mathrm{d} t
$$


Combining (19)-(21), we have

$$
\begin{aligned}
& \int_{I}\left\{(\bar{x}-x)^{\mathrm{T}}\left(r f_{x}+y^{\mathrm{T}} g_{x}+z h_{x}-z\right)\right. \\
& \left.+(u-\bar{u})^{\mathrm{T}}\left(r f_{u}+y^{\mathrm{T}} g_{u}+z^{\mathrm{T}} h_{u}\right)\right\}<0
\end{aligned}
$$

From (13) and (14), we get

$$
\begin{aligned}
& \int_{I}\left\{(\bar{x}-x)^{\mathrm{T}}\left(r f_{x}+y^{\mathrm{T}} g_{x}+z h_{x}-z\right)\right\} \mathrm{d} t \\
& +\int_{I}\left\{(u-\bar{u})^{\mathrm{T}}\left(r f_{u}+y^{\mathrm{T}} g_{u}+z^{\mathrm{T}} h_{u}\right)\right\} \mathrm{d} t=0 \\
& \int_{I}\left\{(\bar{x}-x)^{\mathrm{T}}\left(r f_{x}+y^{\mathrm{T}} g_{x}+z h_{x}-z\right)\right. \\
& \left.\quad+(u-\bar{u})^{\mathrm{T}}\left(r f_{u}+y^{\mathrm{T}} g_{u}+z^{\mathrm{T}} h_{u}\right)\right\}=0
\end{aligned}
$$

The relation (22) and (23) are in contradiction, thus

$$
\int_{I} f(t, \bar{x}, \bar{u}) \mathrm{d} t \geq \int_{I} f(t, x, u) \mathrm{d} t
$$

Implying

$$
\inf (C P)>\sup \left(F_{r} C D\right) \text {. }
$$

Theorem 3 (Strong Duality): If $(\bar{x}, \bar{u})$ is an optimal solution of (CP), then there exist $r \in R$ and piecewise smooth $y: I \rightarrow R^{m}$ and $z: I \rightarrow R^{k}$ such that $(\bar{x}, \bar{u}, r, y, z)$ is feasible for $\left(\mathrm{F}_{\mathrm{r}} \mathrm{CD}\right)$ and objective values are equal. If hypotheses of Theorem 2 hold, then $(\bar{x}, \bar{u}, r, y, z)$ is an optimal solution of $\left(\mathrm{F}_{\mathrm{r}} \mathrm{CD}\right)$.

Proof: Since $(\bar{x}, \bar{u})$ is an optimal solution of (CP) by Proposition 1, there exist $r \in R$, piecewise smooth $y: I \rightarrow R^{m}$ and $z: I \rightarrow R^{k}$ such that

$$
\begin{gathered}
r f_{x}+y^{\mathrm{T}} g_{x}+z^{\mathrm{T}} h_{x}=z, t \in I \\
r f_{u}+y^{\mathrm{T}} g_{u}+z^{\mathrm{T}} h_{u}=0, t \in I \\
y(t)^{\mathrm{T}} g(t, \bar{x}, \bar{u})=0, t \in I \\
g(t, \bar{x}, \bar{u}) \leq 0, t \in I \\
h(t, \bar{x}, \bar{u})=0, t \in I \\
(r, y(t)) \geq 0, t \in I \\
(r, \bar{y}(t), \bar{z}(t)) \geq 0, t \in I
\end{gathered}
$$

The relation (26) implies

$$
\int_{I} y(t)^{\mathrm{T}} g(t, \bar{x}, \bar{u}) \mathrm{d} t=0
$$

and the relation (28) along $y(t) \geq 0, t \in I$ gives

$$
\int_{I} z(t)^{\mathrm{T}} h(t, \bar{x}, \bar{u}) \mathrm{d} t=0
$$

The relation (24), (25), (29)-(32), yields the feasibility of $(r, \bar{x}, \bar{u}, \bar{y}, \bar{z})$ for $\left(\mathrm{F}_{\mathrm{r}} \mathrm{CD}\right)$. Equality of objective func- tionals of $(\mathrm{CP})$ and $\left(\mathrm{F}_{\mathrm{r}} \mathrm{CD}\right)$ is obvious from their formulations.

Consequently the optimality for $\left(\mathrm{F}_{\mathrm{r}} \mathrm{CD}\right)$ follows, given the pseudo-convexity of the $\int f \mathrm{~d} t$, semi-strict pseudoconvexity of $\int_{I} y^{\mathrm{T}} g \mathrm{~d} t$, and quasí-convexity of $\int_{I} z(t)^{\mathrm{T}}(h-x) \mathrm{d} t$, by Theorem 2.

Theorem 4 (Strict-Converse duality): Assume that

$\left(\mathrm{A}_{1}\right): \int f \mathrm{~d} t$ is strictly pseudo-convex, $\int y(t)^{\mathrm{T}} g \mathrm{~d} t$ is semi-strictly pseudo-convex and $\int z(t)^{\mathrm{T}}(h-x) \mathrm{d} t$ is quasi-convex.

$\left(\mathrm{A}_{2}\right):\left(x_{0}, u_{0}\right)$ is an optimal solution of (CP) and $\left(\mathrm{A}_{3}\right):(\bar{x}, \bar{u}, \bar{r}, \bar{y}, \bar{z})$ is an optimal solution of $\left(\mathrm{F}_{\mathrm{r}} \mathrm{CD}\right)$.

Then $(\bar{x}, \bar{u})$ is an optimal solution of (CP) with $\left(x_{0}, u_{0}\right)=(\bar{x}, \bar{u}), t \in I$.

Proof: we suppose $\left(x_{0}, u_{0}\right) \neq(\bar{x}, \bar{u})$ and exhibit a contradiction. Since $\left(x_{0}, u_{0}\right)$ is an optimal solution of (CP) by theorem (Strong Duality) that there exist

$(\bar{r}, \bar{y}(t), \bar{z}(t)), t \in I$ where $r \in R$ and piecewise smooth $y: I \rightarrow R^{m}$ and piecewise smooth $y: I \rightarrow R^{m}$ and $z: I \rightarrow R^{k}$ such that $(\bar{x}, \bar{u}, \bar{r}, \bar{y}, \bar{z})$ is also an optimal solution for (FrCD), it follows that

$$
\int_{I} f\left(t, x_{0}, u_{0}\right) \mathrm{d} t \geq \int_{I} f(t, \bar{x}, \bar{u}) \mathrm{d} t
$$

By strict pseudo-convexity of $\int_{I} \mathrm{f} \mathrm{d} t$ gives, this implies

$$
\int_{I}\left\{\left(x_{0}-\bar{x}\right)^{\mathrm{T}} f_{x}+\left(u_{0}-\bar{u}\right)^{\mathrm{T}} f_{u}\right\} \mathrm{d} t<0
$$

and multiplying the above by $r \geq 0$

$$
\int_{I}\left\{\left(x_{0}-\bar{x}\right)^{\mathrm{T}} r f_{x}+\left(u_{0}-\bar{u}\right)^{\mathrm{T}} r f_{u}\right\} \mathrm{d} t \leq 0
$$

with strict inequality if $r>0$. From the constraints of (CP) and (FrCD), we have

$$
\int_{I} y(t)^{\mathrm{T}} g\left(t, x_{0}, u_{0}\right) \mathrm{d} t \leq \int_{I} y(t) g(t, \bar{x}, \bar{u}) \mathrm{d} t
$$

Also

$$
\begin{aligned}
& \int_{I} z(t)^{\mathrm{T}}\left(h\left(t, x_{0}, u_{0}\right)-\bar{x}_{0}\right) \mathrm{d} t \\
& \leq \int_{I} z(t)^{\mathrm{T}}(h(t, \bar{x}, \bar{u})-\bar{x}) \mathrm{d} t
\end{aligned}
$$

By semi-strict pseudoconvexity of $\int_{I} y(t)^{\mathrm{T}} g \mathrm{~d} t$ and from (34), we have

$$
\int_{I}\left\{\left(x_{0}-\bar{x}\right)^{\mathrm{T}}\left(y^{\mathrm{T}} g_{x}\right)+\left(u_{0}-\bar{u}\right)^{\mathrm{T}}\left(y^{\mathrm{T}} g_{u}\right)\right\} \mathrm{d} t \leq 0
$$

with strict inequality in the above if,

$$
y^{i}(t)>0, t \in I, i \in\{1,2,3, \quad, m\}
$$


By quasi-convexity of $\int_{I} z(t)^{\mathrm{T}}(h-x) \mathrm{d} t$, and from (35), we get

$$
\begin{array}{r}
\int_{I}\left\{\left(x_{0}-\bar{x}\right)\left(z h_{x}\right)-\left(x_{0}-\bar{x}\right) z(t)\right. \\
\left.+\left(u_{0}-\bar{u}\right)^{\mathrm{T}}\left(z^{\mathrm{T}}(t) h_{u}\right)\right\} \mathrm{d} t \leq 0
\end{array}
$$

As earlier, this reduces to

$$
\begin{aligned}
& \int_{I}\left\{\left(x_{0}-\bar{x}\right)\left(z(t)^{\mathrm{T}} h_{x}-z(t)\right)\right. \\
& \left.+\left(u_{0}-\bar{u}\right)^{\mathrm{T}}\left(z(t) h_{u}\right)\right\} \mathrm{d} t \leq 0
\end{aligned}
$$

combining (33), (36), and (37), we have

$$
\begin{aligned}
& \int_{I}\left\{\left(x_{0}-\bar{x}\right)\left(r f_{x}+y^{\mathrm{T}} g_{x}+z(t)^{\mathrm{T}}(h-x)\right)\right. \\
& \left.\quad+\left(u_{0}-\bar{u}\right)^{\mathrm{T}}\left(r f_{u}+y(t)^{\mathrm{T}} g_{u}+z(t)^{\mathrm{T}} h_{u}\right)\right\} \mathrm{d} t<0
\end{aligned}
$$

$$
M(t)=\left(\begin{array}{cc}
r f_{x x}+y(t)^{\mathrm{T}} g_{x x}+z(t)^{\mathrm{T}} h_{x x} & r f_{x u}+y(t)^{\mathrm{T}} g_{x u}+z(t)^{\mathrm{T}} h_{x u} \\
r f_{u x}+y^{\mathrm{T}} g_{u x}+z h_{u x} & r f_{u u}+y(t)^{\mathrm{T}} g_{u u}+z(t)^{\mathrm{T}} h_{u u}
\end{array}\right)
$$

and $\left(\mathrm{A}_{4}\right) \quad z(a)=0=z(b)$

Then $(\bar{x}, \bar{u})$ is optimal for (CP).

Proof: By Proposition 1, there exist $\tau \in R, \alpha \in R$, $\beta \in R, \xi \in R$, piecewise smooth $\theta: I \rightarrow R^{m}$ and $\phi: I \rightarrow R^{n}$ such that

$$
\begin{gathered}
\tau f_{x}+\theta(t)^{\mathrm{T}}\left(r f_{x x}+y(t)^{\mathrm{T}} g_{x x}+z(t)^{\mathrm{T}} h_{x x}\right) \\
+\phi(t)^{\mathrm{T}}\left(r f_{u x}+y(t)^{\mathrm{T}} g_{u x}+z(t)^{\mathrm{T}} h_{u x}\right) \\
+\alpha\left(y(t)^{\mathrm{T}} g_{x}\right)+\beta\left(z(t)^{\mathrm{T}} h_{x}-z(t)\right)=0, t \in I \\
\tau f_{u}+\theta(t)^{\mathrm{T}}\left(r f_{u x}+y(t)^{\mathrm{T}} g_{u x}+z(t)^{\mathrm{T}} h_{u x}\right) \\
+\phi(t)^{\mathrm{T}}\left(r f_{u u}+y(t)^{\mathrm{T}} g_{u u}+z(t)^{\mathrm{T}} h_{u u}\right)=0, t \in I \\
\theta(t)^{\mathrm{T}} f_{x}+\phi(t)^{\mathrm{T}} f_{u}+\xi=0 \\
\theta(t)^{\mathrm{T}} h_{x}+\phi(t)^{\mathrm{T}} h_{u}+\beta(h-x)+\theta(t)=0, t \in I \\
\alpha g_{I}+\phi(t)^{\mathrm{T}} g \mathrm{~d} t=0 \\
\beta \int_{I} z(t)^{\mathrm{T}}(h-x) \mathrm{d} t=0 \\
\xi r=0 \\
\eta(t)^{\mathrm{T}} y(t)=0, t \in I \\
(\tau, \alpha, \beta, \xi, \eta(t)) \geq 0, t \in I
\end{gathered}
$$

This contradicts the feasibility of $(\bar{x}, \bar{u}, r, y, z)$ for ( $\left.\mathrm{F}_{\mathrm{r}} \mathrm{CD}\right)$, hence $(\bar{x}, \bar{u})$ is an optimal solution of (CP) and $(\bar{x}, \bar{u})=\left(x_{0}, u_{0}\right)$.

Theorem 5 (Converse duality): Let $(x, r, y, z)$ be an optimal solution of $\left(\mathrm{F}_{\mathrm{r}} \mathrm{CD}\right)$, Assume

$\left(\mathrm{A}_{1}\right) \int_{I} f(t) \mathrm{d} t$ is pseudo-convex, $\int_{I} y(t)^{\mathrm{T}} g \mathrm{~d} t$ is semi-strictly pseudo-convex and $\int_{I} z^{\mathrm{T}}(h-x) \mathrm{d} t$ is quasiconvex.

$\left(\mathrm{A}_{2}\right)$ The set $\left\{y(t)^{\mathrm{T}} g_{x}, z(t)^{\mathrm{T}} h_{x}-z(t)\right\}$ or $\left\{y(t)^{\mathrm{T}} g_{u}, z(t)^{\mathrm{T}} h_{u}\right\}$ is linearly independent.

$\left(\mathrm{A}_{3}\right) \int_{I} v(t)^{\mathrm{T}} M(t) v(t) \mathrm{d} t=0, \Rightarrow v(t)=0, t \in I$, for some column vector $v(t)$ and where

$$
(\tau, \alpha, \beta, \xi, \theta(t), \phi(t), \eta(t)) \neq 0, t \in I
$$

Multiplying (41) by $y(t)$ and integrating, and then using (43) and (46), we have

$$
\int_{I}\left\{\theta(t)^{\mathrm{T}}\left(y(t)^{\mathrm{T}} g_{x}\right)+\phi(t)^{\mathrm{T}}\left(y(t)^{\mathrm{T}} g_{u}\right)\right\} \mathrm{d} t=0
$$

which can be written as

$$
\int_{I}(\theta(t), \phi(t))\left(\begin{array}{ll}
y(t)^{\mathrm{T}} & g_{x} \\
y(t)^{\mathrm{T}} & g_{u}
\end{array}\right) \mathrm{d} t=0
$$

Multiplying (42) by $z(t)$, and then integrating we get

$$
\begin{aligned}
& \int_{I}\left(\theta(t)^{\mathrm{T}}\left(z(t)^{\mathrm{T}} h_{x}\right)+\phi(t)^{\mathrm{T}}\left(z(t)^{\mathrm{T}} h_{u}\right)\right) \mathrm{d} t \\
& +\int_{I} \theta(t) z(t)^{\mathrm{T}} \mathrm{d} t+\beta \int_{I} z(t)^{\mathrm{T}}(h-x) \mathrm{d} t=0, t \in I,
\end{aligned}
$$

where $\theta=\frac{\mathrm{d} \theta}{\mathrm{d} t}$ and $x=\frac{\mathrm{d} x}{\mathrm{~d} t}$, using (44), this yields

$$
\begin{aligned}
& 0=\int_{I}\left(\theta(t)^{\mathrm{T}}\left(z(t)^{\mathrm{T}} h_{x}\right)+\phi(t)^{\mathrm{T}}\left(z(t)^{\mathrm{T}} h_{u}\right)\right) \mathrm{d} t \\
& \quad+\int_{I} \theta(t) z(t)^{\mathrm{T}} \mathrm{d} t, t \in I \\
& 0=\int_{I}\left(\theta(t)^{\mathrm{T}}\left(z(t)^{\mathrm{T}} h_{x}\right)+\phi(t)^{\mathrm{T}}\left(z(t)^{\mathrm{T}} h_{u}\right)\right) \mathrm{d} t \\
& \quad+\left.z(t) \theta(t)\right|_{t=a} ^{t=b}-\int_{I} \theta(t) z(t) \mathrm{d} t
\end{aligned}
$$

(by integrating by parts)

which in view of $\left(\mathrm{A}_{4}\right)$, implies 


$$
\int_{I}\left[\theta(t)^{\mathrm{T}}\left(z(t)^{\mathrm{T}} h_{x}-z(t)\right)+\phi(t)^{\mathrm{T}}\left(z(t)^{\mathrm{T}} h_{u}\right)\right] \mathrm{d} t=0
$$

This can be written as

$$
\int_{I}\left(\theta(t)^{\mathrm{T}}, \phi(t)^{\mathrm{T}}\right)\left(\begin{array}{c}
z(t)^{\mathrm{T}} h_{x}-z(t) \\
z(t)^{\mathrm{T}} h_{u}
\end{array}\right) \mathrm{d} t=0
$$

$$
\begin{aligned}
& (r \alpha-\tau)\left(y(t)^{\mathrm{T}} g_{x}\right)+(r \beta-\tau)\left(z(t)^{\mathrm{T}} h_{x}-z(t)\right) \\
& +r \theta(t)^{\mathrm{T}}\left(r f_{x x}+y(t)^{\mathrm{T}} g_{x x}+z(t)^{\mathrm{T}} h_{x x}\right) \\
& +r \phi(t)\left(r f_{u x}+y(t)^{\mathrm{T}} g_{u x}+z(t)^{\mathrm{T}} h_{u x}\right)=0
\end{aligned}
$$

These can be combined as

$$
\begin{aligned}
& (r \alpha-\tau)\left(y(t)^{\mathrm{T}} g_{u}\right)+(r \beta-\tau)\left(z(t)^{\mathrm{T}} h_{u}\right)+r \theta(t)^{\mathrm{T}}\left(r f_{u x}+y(t)^{\mathrm{T}} g_{u x}+z(t)^{\mathrm{T}} h_{u x}\right) \\
& +r \phi(t)\left(r f_{u u}+y(t)^{\mathrm{T}} g_{u u}+z(t)^{\mathrm{T}} h_{u u}\right)=0 \\
& (r \alpha-\tau)\left(\begin{array}{c}
y(t)^{\mathrm{T}} g_{x} \\
y(t)^{\mathrm{T}} g_{u}
\end{array}\right)+(r \beta-\tau)\left(\begin{array}{c}
z(t)^{\mathrm{T}} h_{x}-z(t) \\
z(t)^{\mathrm{T}} h_{u}
\end{array}\right) \\
& +\left(\begin{array}{cc}
r f_{x x}+y(t)^{\mathrm{T}} g_{x x}+z(t)^{\mathrm{T}} h_{x x} & r f_{u x}+y(t)^{\mathrm{T}} g_{u x}+z(t)^{\mathrm{T}} h_{u x} \\
r f_{u x}+y(t)^{\mathrm{T}} g_{u x}+z(t)^{\mathrm{T}} h_{u x} & r f_{u u}+y(t)^{\mathrm{T}} g_{u u}+z(t)^{\mathrm{T}} h_{u u}
\end{array}\right) \times\left(\begin{array}{c}
r \theta(t) \\
r \phi(t)
\end{array}\right)=0
\end{aligned}
$$

Pre-multiplying this by $\left(r \theta(t)^{\mathrm{T}}, r \phi(t)^{\mathrm{T}}\right)$ and then integrating we have

$$
\begin{aligned}
& (r \alpha-\tau) \int_{I}\left(r \theta(t)^{\mathrm{T}}, r \phi(t)^{\mathrm{T}}\right)\left(\begin{array}{c}
y(t)^{\mathrm{T}} g_{x} \\
y(t)^{\mathrm{T}} g_{u}
\end{array}\right) \mathrm{d} t+(r \beta-\tau) \int_{I}\left(r \theta(t)^{\mathrm{T}}, r \phi(t)^{\mathrm{T}}\right)\left(\begin{array}{c}
z(t)^{\mathrm{T}} h_{x}-z(t) \\
z(t)^{\mathrm{T}} h_{u}
\end{array}\right) \mathrm{d} t \\
& +\int_{I}\left\{\left(r \theta(t)^{\mathrm{T}}, r \phi(t)^{\mathrm{T}}\right) \times\left(\begin{array}{cc}
r f_{x x}+y(t)^{\mathrm{T}} g_{x x}+z(t)^{\mathrm{T}} h_{x x} & r f_{u x}+y(t)^{\mathrm{T}} g_{u x}+z(t)^{\mathrm{T}} h_{u x} \\
r f_{u x}+y(t)^{\mathrm{T}} g_{u x}+z(t)^{\mathrm{T}} h_{u x} & r f_{u u}+y(t)^{\mathrm{T}} g_{u u}+z(t)^{\mathrm{T}} h_{u u}
\end{array}\right) \times(r \theta(t), r \phi(t))\right\} \mathrm{d} t=0
\end{aligned}
$$

Using (49) and (50), this implies

$$
\int_{I}\left\{\left(r \theta(t)^{\mathrm{T}}, r \phi(t)^{\mathrm{T}}\right) \times\left(\begin{array}{ll}
r f_{x x}+y(t)^{\mathrm{T}} g_{x x}+z(t)^{\mathrm{T}} h_{x x} & r f_{u x}+y(t)^{\mathrm{T}} g_{u x}+z(t)^{\mathrm{T}} h_{u x} \\
r f_{u x}+y(t)^{\mathrm{T}} g_{u x}+z(t)^{\mathrm{T}} h_{u x} & r f_{u u}+y(t)^{\mathrm{T}} g_{u u}+z(t)^{\mathrm{T}} h_{u u}
\end{array}\right) \times(r \theta(t), r \phi(t))\right\} \mathrm{d} t=0
$$

which in view of $\left(\mathrm{A}_{2}\right)$ implies

$v(t)=(r \theta(t), r \phi(t))=0, t \in I$ implies

$$
r \theta(t)=0, r \phi(t)=0, t \in I
$$

In view of $\left(\mathrm{A}_{3}\right)$, the equality constraint implies $r \neq 0$ i.e. $r>0$. Consequently, we have

$$
\theta(t)=0, t \in I, \phi(t)=0, t \in I,
$$

Using (52), along with $r>0$, we have

$$
\left(\alpha-\frac{\tau}{r}\right)\left(y(t)^{\mathrm{T}} g_{x}\right)+\left(\beta-\frac{\tau}{r}\right)\left(z(t)^{\mathrm{T}} h_{x}-z(t)\right)=0
$$

This, in view of $\left(\mathrm{A}_{3}\right)$,

$$
\alpha-\frac{\tau}{r}=0, \beta-\frac{\tau}{r}=0
$$

If $\tau=0$, (53), implies $\alpha=0=\beta$. Thus $(\tau, \alpha, \beta, \xi, \theta(t), \phi(t), \eta(t))=0$, contradiction.
Hence $\tau>0$ and consequently $\alpha>0$ and $\beta>0$. From (41) and (42), we have

$$
g(t, x, u) \leq 0, t \in I, h(t, x, u)=x, t \in I
$$

These relations yield the feasibility of $(\bar{x}, \bar{u})$ for (CP) and objective functionals of (CP) and (FrCD) are equal there. Hence under the stated convexity hypotheses, by Theorem 2, $(\bar{x}, \bar{u})$ is an optimal solution of (CP).

\section{Mathematical Programming Problems}

If the problems (CP) and $\left(\mathrm{F}_{\mathrm{r}} \mathrm{CD}\right)$ are independent of $t$ and $\mathrm{x}$, these problems reduce to essentially to the static cases of nonlinear programming problem. Letting $b-a=1$, the problems (CP) and ( $\mathrm{F}_{\mathrm{r}} \mathrm{CD}$ ) become the pair of dual nonlinear programming problems formulated by Husain and Srivastav [9].

$\left(\mathrm{CD}_{0}\right)$ : Minimize $f(u)$ subject to 


$$
\begin{aligned}
& g(u) \leq 0, \\
& h(u)=0 .
\end{aligned}
$$

(FrCD): Maximize $f(u)$ subject to

$$
\begin{aligned}
r f_{u}(u)+y^{\mathrm{T}} g_{u}(u)+z^{\mathrm{T}} h_{u}(u) & =0, \\
y^{\mathrm{T}} g(u) & \geq 0, \\
z^{\mathrm{T}} h(u) & \geq 0, \\
(r, y) & \geq 0, \\
(r, y, z) & \neq 0 .
\end{aligned}
$$

where $f$ is pseudoconvex, $y^{\mathrm{T}} g(\cdot)$ is semi-strictly pseudoconvex and $z^{\mathrm{T}} h$ is quasi-convex. If only inequality constraint in $\left(\mathrm{CD}_{0}\right)$ is given, then $\left(\mathrm{CP}_{0}\right)$ and $\left(\mathrm{F}_{\mathrm{r}} \mathrm{CD}_{0}\right)$ become a pair of dual the nonlinear programming problems considered by Weir and Mond [10].

\section{Conclusion}

In this paper, sufficient optimality conditions are derived for a control problem which appears in various real life situations under generalized convexity assumptions. In order to formulate the dual to this control problem, Fritz John optimality conditions are used instead of KarushKuhn-Tucker optimality condition and hence the requirement of regularity condition is eliminated. Various duality results are obtained and the linkage of our duality results to those of a nonlinear programming problem is indicated. Our results can be seen in the setting of multiobjective control problems.

\section{Acknowledgements}

The authors acknowledge anonymous referees for their valuable comments which have improved the presentation of this research paper.

\section{REFERENCES}

[1] L. D. Berkovitz, "Variational Methods in Problems of Control and Programming," Journal of Mathematical Analysis and Applications, Vol. 3, No. 1, 1961, pp. 145169. doi:10.1016/0022-247X(61)90013-0

[2] B. Mond and M. A. Hanson, "Duality for Control Problems,” SIAM Journal on Control, Vol. 6, 1965, pp. 28-35.

[3] S. Chandra, B. D. Craven and I. Husain "A Class of Nondifferentiable Control Problems,” Journal of Optimization Theory and Applications, Vol. 56, No. 2, 1988, pp. 227243. doi:10.1007/BF00939409

[4] B. D. Craven, "Mathematical Programming and Control Theory," Chapman and Hall, London, 1978. doi:10.1007/978-94-009-5796-1

[5] F. A. Valentine, “The Problem of Lagrange with Differential Inequalities as Added Side Conditions, Contribution to the Calculus of Variation, 1933-1937," University of Chicago Press, Chicago, 1937, pp. 407-448.

[6] B. Mond and I. Smart, "Duality and Sufficiency in Control Problems with Invexity," Journal of Mathematical Analysis and Applications, Vol. 136, No. 15, 1988, pp. 325-333.

[7] I. Husain, A. Ahmed and B. Ahmad, "Sufficiency and Duality in Control Problems with Generalized Invexity," Journal of Applied Analysis, Vol. 14 No. 1, 2008, pp. $27-$ 42. doi:10.1515/JAA.2008.27

[8] B. Mond and T. Weir, "Generalized Concavity and Duality in Generalized Concavity in Optimization and Economics,” In: S. Schaibl and W. T. Ziemba, Eds., Academic Press, New York, 1981, pp. 263-279.

[9] I. Husain and S. K. Shrivastav, "Fritz John Duality in the Presence of Equality and Inequality Constarints,” Applied Mathematics, Vol. 3, No. 9, 2012, pp. 1023-1028. doi:10.4236/am.2012.39151

[10] T. Weir and B. Mond, "Sufficient Fritz John Optimality Conditions and Duality for Nonlinear Programming Problems,” Opsearch, Vol. 23, No. 3, 1986, pp. 129-141. 